
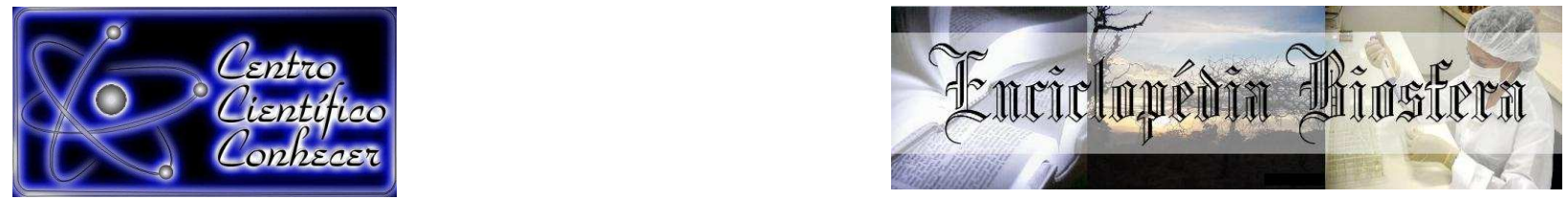

\title{
DETERMINAÇÃO DA TAXA DE INFILTRAÇÃO DE ÁGUA NO SOLO E LÂMINA INFILTRADA EM LATOSSOLO VERMELHO-AMARELO
}

Dionei Lima Santos ${ }^{1}$, Sidney Geraldo Silveira Velloso², José Geraldo de Araújo Ferreira Filho ${ }^{3}$

1- Engenheiro Agrônomo, Mestrando do Programa de Pós-Graduação em Engenharia Agrícola da Universidade Federal de Viçosa, Viçosa/MG, Brasil dionei_lima12@hotmail.com

2- Engenheiro Florestal, Mestrando do Programa de Pós-Graduação em Ciência Florestal da Universidade Federal de Viçosa

3- Engenheiro Agrícola, Mestrando do Programa de Pós-Graduação em Engenharia

Agrícola da Universidade Federal de Viçosa

Recebido em: 08/09/2015 - Aprovado em: 14/11/2015 - Publicado em: 01/12/2015 DOI: http://dx.doi.org/10.18677/Enciclopedia_Biosfera_2015_134

\section{RESUMO}

O processo de infiltração de água no solo consiste na passagem de água, através da superfície do mesmo, de forma descendente. Modelos empíricos e teóricos tem sido propostos para descreverem a forma como a infiltração se processa. Pode-se citar como modelos empíricos e teóricos comumente encontrados na literatura aqueles propostos por Kostiakov e Kostiakov-Lewis e, Green-Ampt, respectivamente. O objetivo do presente estudo foi ajustar os modelos de Kostiakov, Kostiakov-Lewis e Green-Ampt, a partir de dados observados por meio de ensaio de infiltrômetro de anéis. As equações ajustadas para os modelos de Kostiakov e Kostiakov-Lewis, para taxa de infiltração de água no solo, foram, respectivamente, $i$ $=3,27^{*} t^{-0,3411} i=10,1435^{*} t^{-0,7568}+72$, sendo que tais equações descreveram de forma condizente o processo de infiltração. Os parâmetros obtidos para o modelo de Green-Ampt, a partir de amostras de solo, foram $K_{s}=39,1 \mathrm{~mm} \cdot \mathrm{h}^{-1}, \Psi_{f}=46530,612$ mm.c.a., $\theta_{s}=33,42 \%$ e $\theta_{i}=24,14 \%$, sendo que alterações nestes parâmetros foram propostas, de forma a identificar aquela que melhor se adequasse aos dados observados no ensaio de infiltrômetro de anéis. A combinação que apresentou menor valor de RMSE foi C13, em que $K_{t}=$ Tie, $\theta_{w}=0,86 \theta_{s} \mathrm{e}$ $\Psi_{f}=49,03\left(K_{s}+0,02\right)^{-0,4932}$.

PALAVRAS-CHAVE: Green-Ampt, infiltrômetro de anéis, Kostiakov.

\section{DETERMINATION OF WATER INFILTRATION RATE IN SOIL AND INFILTRATED LAMINA IN YELLOW-REDISH OXYSOIL}

\begin{abstract}
The water infiltration in soil process consists of passing water, through its surface, downwardly. Theoretical and empirical models have been proposed to describe how the infiltration process. It can be cited, as empirical and theoretical models commonly found in literature, those proposed by Kostiakov and Kostiakov-Lewis and, Green-
\end{abstract}


Ampt, respectively. The aim of this study was to adjust Kostiakov, Kostiakov-Lewis and Green-Ampt models, from observed data obtained by infiltrometer rings test. The adjusted equation for Kostiakov and Kostiakov-Lewis models, for water infiltration rate in soil, were, respectively, $i=3,27^{*} t^{-0,3411} i=10,1435^{\star} t^{-0,7568}+72$, and these equations described consistently the infiltration process. The parameters for GreenAmpt model, obtained from soil samples, were $K_{s}=39,1 \mathrm{~mm} \cdot \mathrm{h}^{-1}, \Psi_{f}=46530,612$ mm.c.a., $\theta_{s}=33,42 \%$ e $\theta_{i}=24,14 \%$, and changes in such parameters were proposed, to identify the one that would best fit the data observed in the infiltrometer rings test. The combination that showed the lowest RMSE value was C13, where $K_{t}$ $=$ Tie, $\theta_{w}=0,86 \theta_{s}$ e $\Psi_{f}=49,03\left(K_{s}+0,02\right)^{-0,4932}$.

KEYWORDS: Green-Ampt, infiltrometer rings, Kostiakov.

\section{INTRODUÇÃO}

A infiltração da água no solo consiste na passagem de água, através da superfície do mesmo, em um sentindo descendente. Esta passagem é possível devido ao fato do solo ser um material poroso e, portanto, apresentar um potencial total de condução da água. Este potencial total é obtido pela soma da contribuição dos potenciais matricial (inerente ao solo propriamente dito e função de suas características), osmótico (relativo à presença de uma solução do solo com determinada concentração de solutos), gravitacional (realizado a partir da força de atração gravitacional terrestre) e de pressão (que surge quando da existência de uma lâmina considerável de fluído sobre a superfície do solo). Em situações práticas, como aquelas adotadas em ensaios de campo, os potenciais de pressão e osmótico podem ser desconsiderados (PRUSKI et al., 1997).

A taxa com que a passagem da água sobre a superfície do solo se processa é referida como a taxa de infiltração de água no solo, anteriormente denominada de velocidade de infiltração e, é influenciada por diversos fatores, como condições de superfície, conteúdo inicial de água no perfil do solo, tempo de infiltração, existência de camadas impermeáveis ao longo do perfil e propriedades físicas do solo (BERNARDO, 1986). Dentre estes fatores, destaca-se o selamento superficial causado pelo impacto das gotas de chuva em solos desprotegidos e, que reduz ou impede a passagem de água através de sua superfície. A partir deste adendo, percebe-se a importância do correto manejo da cobertura vegetal para que se aumente a lâmina total infiltrada (MACHADO et al., 2014). O objetivo que procura-se atingir com um aumento na parcela infiltrada a partir do total precipitado diz respeito à permanência da água em bacias hidrográficas, uma vez que a água contida nos mananciais subterrâneos é aquela que vai alimentar os cursos d'água em períodos de seca, bem como fornecer suporte hídrico às plantas durante a estiagem.

Alguns autores buscaram representar a forma com que ocorre o processo de infiltração da água no solo e, descreveram relações matemáticas baseadas no conhecimento adquirido a partir da análise do processo físico, ou a partir do melhor ajuste de determinada equação, referindo-se assim aos modelos teórico e empírico, respectivamente.

Dentre os modelos teóricos, um de grande uso, devido sua simplicidade e embasamento físico, é o modelo apresentado por GREEN \& AMPT (1911) (ZONTA et al., 2012) que, baseados na equação de Darcy, descreveram o processo de infiltração da água no solo a partir de algumas pressuposições, como presença de uma frente de umedecimento abrupta (onde o conteúdo de umidade imediatamente abaixo da frente de umedecimento é igual ao conteúdo de umidade inicial e, o ENCICLOPÉDIA BIOSFERA, Centro Científico Conhecer - Goiânia, v.11 n.22; p. 9582015 
conteúdo de umidade em qualquer ponto acima da frente de umedecimento é igual ao conteúdo de umidade de saturação), potencial matricial na frente de umedecimento constante e igual aquele apresentado pelo solo à umidade inicial, carga hidráulica constante sobre o solo e solo homogêneo com profundidade infinita (CECÍLIO et al., 2003; PRUSKI et al., 1997).

Dentre os modelos empíricos, destaca-se aquele apresentado por KOSTIAKOV (1932) que, a partir da observação do processo de infiltração, modelou-o conforme uma relação exponencial onde o total infiltrado é função do tempo de infiltração e de parâmetros empíricos associados às características do solo em questão. Deve-se ressaltar que o modelo de Kostiakov apresentava a limitação de que, quando o tempo de infiltração se tornasse demasiadamente elevado, a taxa de infiltração tenderia a um valor nulo, sendo que tal fato não ocorre na realidade. Portanto, foi necessária uma modificação na equação proposta, onde uma constante (relacionada à taxa de infiltração estável do solo) foi adicionada por Lewis, culminando no modelo de Kostiakov-Lewis.

Diante do exposto, o objetivo do presente estudo foi ajustar as equações de Kostiakov, Kostiakov-Lewis e Green-Ampt a partir de dados obtidos por meio de ensaio experimental realizado com infiltrômetro de anéis, numa área localizada no campus da Universidade Federal de Viçosa, bem como avaliar a qualidade de tais ajustes.

\section{MATERIAL E MÉTODOS}

O estudo foi realizado em uma área experimental do Centro de Referência em Recursos Hídricos da Universidade Federal de Viçosa. Sob coordenadas 4552'W e 20\%48'S do datum WGS-84, o local onde foi conduzido o ensaio de infiltração de água no solo possui cobertura vegetal gramínea distribuída uniformemente em relevo plano, com predominância de Latossolo Vermelho-Amarelo. A classificação climática de Köppen para a região é do tipo Cwa com verões chuvosos e invernos secos.

As variáveis para ajuste das equações foram obtidas por meio de ensaio de infiltrômetro de anéis concêntricos. Neste método, cravam-se dois anéis de forma concêntrica no solo, onde o anel interno apresenta diâmetro de $250 \mathrm{~mm}$, enquanto o anel externo apresenta diâmetro de $500 \mathrm{~mm}$. Alimentando-se ambos os anéis com água, mede-se a altura de lâmina, no anel interno, em intervalos regulares de tempo.

Os dados obtidos foram utilizados para ajuste dos modelos de Kostiakov, Kostiakov-Lewis e Green-Ampt. As equações de infiltração de água no solo para os modelos de Kostiakov e Kostiakov-Lewis são $l=k^{*} t \alpha$ e $l=k^{*} t+T_{i e} t$, respectivamente, onde l: lâmina total infiltrada, $k$ e $\alpha$ : constantes empíricas, $t$ : intervalo de tempo, $T_{i e}$ : taxa de infiltração estável. A partir da derivada primeira da equação de lâmina infiltrada, obtêm-se as equações de taxa de infiltração para os modelos de Kostiakov, $i=k^{*} \alpha^{*} t^{\alpha-1}$ e Kostiakov-Lewis, $i=k^{*} \alpha^{*} t^{\alpha-1}+T_{i e}$, onde $i$ : taxa de infiltração de água no solo.

Para o modelo de Green-Ampt, a equação de taxa de infiltração de água no solo é $i=K_{t}{ }^{*}\left(1+\Psi_{f}^{*}\left(\left(\theta_{w}-\theta_{i}\right)^{*} \Gamma^{-1}\right)\right)$, onde $K_{t}$ : condutividade hidráulica do solo saturado em condições de campo, $\Psi_{f}$ : potencial matricial do solo na frente de umedecimento, $\theta_{w}$ : teor de umidade do solo saturado em condições de campo e, $\theta_{i}$ : teor de umidade inicial do solo. Como são assumidas algumas pressuposições neste modelo, foram determinados os parâmetros $K_{s}, \Psi_{f}, \theta_{s}$ e $\theta_{i}$, a partir de amostras de solo coletadas e utilizadas em métodos de análise e determinação de tais parâmetros, onde $K_{s}$ e $\theta_{s}$ 
são a condutividade hidráulica do solo saturado e umidade do solo saturado, respectivamente. Para determinação dos teores de umidade inicial e do solo saturado, foi utilizado o método gravimétrico; para condutividade hidráulica do solo saturado, foi utilizada a recomendação estabelecida por EMBRAPA (1997) e; para determinação do potencial matricial na frente de umedecimento foi obtida a curva de retenção de água no solo.

Como as pressuposições para utilização do modelo de Green-Ampt muitas vezes não condizem com a realidade, algumas modificações quanto aos parâmetros do mesmo comumente são adotadas. Neste estudo, foram combinadas variações nos parâmetros $\theta_{w}, \Psi_{f}$ e $K_{t}$, conforme apresentado na tabela 1.

TABELA 1: Combinações adotadas para melhor ajuste dos parâmetros do modelo de Green-Ampt às condições de campo.

\begin{tabular}{|c|c|c|c|}
\hline Combinação & $K_{t}$ & $\Psi_{f}$ & $\theta_{w}$ \\
\hline C1 & $T_{i e}$ & $\Psi_{f}=\frac{\Psi\left(\theta_{s}\right)+\Psi\left(\theta_{i}\right)}{2}$ & $0,86 \times \theta_{s}$ \\
\hline $\mathrm{C2}$ & $T_{i e}$ & $\Psi_{f}=\frac{\Psi\left(\theta_{s}\right)+\Psi\left(\theta_{i}\right)}{2}$ & $0,90 \times \theta_{s}$ \\
\hline $\mathrm{C3}$ & $T_{i e}$ & $\Psi_{f}=\frac{\Psi\left(\theta_{s}\right)+\Psi\left(\theta_{i}\right)}{2}$ & $0,84 \times \theta_{s}$ \\
\hline C4 & $T_{i e}$ & $\Psi_{f}=\frac{\Psi\left(\theta_{s}\right)+\Psi\left(\theta_{i}\right)}{2}$ & $0,97 \times \theta_{s}$ \\
\hline C5 & $T_{i e}$ & $\Psi_{f}=\frac{\Psi\left(\theta_{s}\right)+\Psi\left(\theta_{i}\right)}{2}$ & $0,92 \times \theta_{s}$ \\
\hline C6 & $T_{i e}$ & $\Psi_{f}=\frac{\Psi\left(\theta_{s}\right)+\Psi\left(\theta_{i}\right)}{2}$ & $0,79 \times \theta_{s}$ \\
\hline C7 & $K_{s}$ & $\Psi_{f}=\frac{\Psi\left(\theta_{s}\right)+\Psi\left(\theta_{i}\right)}{2}$ & $0,86 \times \theta_{s}$ \\
\hline C8 & $K_{s}$ & $\Psi_{f}=\frac{\Psi\left(\theta_{s}\right)+\Psi\left(\theta_{i}\right)}{2}$ & $0,90 \times \theta_{s}$ \\
\hline C9 & $K_{s}$ & $\Psi_{f}=\frac{\Psi\left(\theta_{s}\right)+\psi\left(\theta_{i}\right)}{2}$ & $0,84 \times \theta_{s}$ \\
\hline C10 & $K_{s}$ & $\Psi_{f}=\frac{\Psi\left(\theta_{s}\right)+\Psi\left(\theta_{i}\right)}{2}$ & $0,97 \times \theta_{s}$ \\
\hline C11 & $K_{s}$ & $\Psi_{f}=\frac{\Psi\left(\theta_{g}\right)+\Psi\left(\theta_{i}\right)}{2}$ & $0,92 \times \theta_{s}$ \\
\hline C12 & $K_{s}$ & $\Psi_{f}=\frac{\Psi\left(\theta_{s}\right)+\Psi\left(\theta_{i}\right)}{2}$ & $0,79 \times \theta_{s}$ \\
\hline C13 & $T_{i e}$ & $\Psi_{f}=49,03\left(K_{s}+0,02\right)^{-0,4932}$ & $0,86 \times \theta_{s}$ \\
\hline C14 & $T_{i e}$ & $\Psi_{f}=49,03\left(K_{s}+0,02\right)^{-0,4932}$ & $0,90 \times \theta_{s}$ \\
\hline C15 & $T_{i e}$ & $\Psi_{f}^{\prime}=49,03\left(K_{g}+0,02\right)^{-0.4932}$ & $0,84 \times \theta_{s}$ \\
\hline C16 & $T_{i e}$ & $\Psi_{f}=49,03\left(K_{s}+0,02\right)^{-0,4932}$ & $0,97 \times \theta_{s}$ \\
\hline C17 & $T_{i e}$ & $\Psi_{f}=49,03\left(K_{z}+0,02\right)^{-0,4932}$ & $0,92 \times \theta_{s}$ \\
\hline C18 & $T_{i e}$ & $\Psi_{f}=49,03\left(K_{s}+0,02\right)^{-0,4932}$ & $0,79 \times \theta_{s}$ \\
\hline C19 & $K_{s}$ & $\Psi_{f}=49,03\left(K_{s}+0,02\right)^{-0,4932}$ & $0,86 \times \theta_{s}$ \\
\hline $\mathrm{C2O}$ & $K_{s}$ & $\Psi_{f}=49,03\left(K_{s}+0,02\right)^{-0,4932}$ & $0,90 \times \theta_{s}$ \\
\hline
\end{tabular}




$\begin{array}{lccc}\text { C21 } & K_{s} & \Psi_{f}=49,03\left(K_{s}+0,02\right)^{-0,4932} & 0,84 \times \theta_{s} \\ \text { C22 } & K_{s} & \Psi_{f}=49,03\left(K_{s}+0,02\right)^{-0,4932} & 0,97 \times \theta_{s} \\ \text { C23 } & K_{s} & \Psi_{f}=49,03\left(K_{s}+0,02\right)^{-0,4932} & 0,92 \times \theta_{s} \\ \text { C24 } & K_{s} & \Psi_{f}=49,03\left(K_{s}+0,02\right)^{-0,4932} & 0,79 \times \theta_{s} \\ \text { C25 } & K_{s} & \Psi_{f}=49,03\left(K_{s}+0,02\right)^{-0,4932} & 0,76 \times \theta_{s} \\ \text { C26 } & K_{s} & \Psi_{f}=\frac{\Psi\left(\theta_{s}\right)+\Psi\left(\theta_{i}\right)}{2} & 0,76 \times \theta_{s} \\ \text { C27 } & T_{i e} & \Psi_{f}=49,03\left(K_{s}+0,02\right)^{-0,4932} & 0,76 \times \theta_{s} \\ \text { C28 } & T_{i e} & \Psi_{f}=\frac{\Psi\left(\theta_{s}\right)+\Psi\left(\theta_{i}\right)}{2} & 0,76 \times \theta_{s}\end{array}$

Por fim, o valor de RMSE (Root Mean Square Error), ou raiz quadrada do erro médio, possibilitou a determinação da combinação que melhor se ajustou aos dados experimentais.

\section{RESULTADOS E DISCUSSÃO}

O ensaio de infiltração de água no solo, por meio do infiltrômetro de anéis concêntricos, teve duração total de 4,12 horas ou 247 minutos. A taxa de infiltração estável foi detectada a partir de 2,87 horas após o início do ensaio, sendo sua magnitude igual a $72 \mathrm{~mm} \cdot \mathrm{h}^{-1}$. VILARINHO et al. (2013), ao determinarem a taxa de infiltração em Latossolo Vermelho Distrófico sob vegetação de cerrado nativo no estado de Mato Grosso, por meio de ensaio de infiltrômetro de anéis, encontraram uma taxa de infiltração estável da magnitude de $36 \mathrm{~cm} \cdot \mathrm{h}^{-1}$. ESPÍRITO SANTO (2011), encontrou valores de 963 e $425 \mathrm{~mm} \cdot \mathrm{h}^{-1}$ para Latossolos Vermelho e Vermelho-Amarelo, respectivamente, sob condições de pastagem na Estação Experimental da Universidade de Brasília. As discrepâncias encontradas entre os valores apresentados, provavelmente estão relacionadas, segundo KAISER (2010), à estrutura do solo. Tais resultados, em conjunto àquele encontrado no presente estudo, demonstram ainda, a maior capacidade de infiltração de água no solo que a cobertura florestal promove em relação à condição de pastagem. As figuras 1 e 2 a seguir apresentam a infiltração de água no tempo e taxa de infiltração de água no tempo, respectivamente, obtidas pelo ensaio de infiltrômetro de anéis.

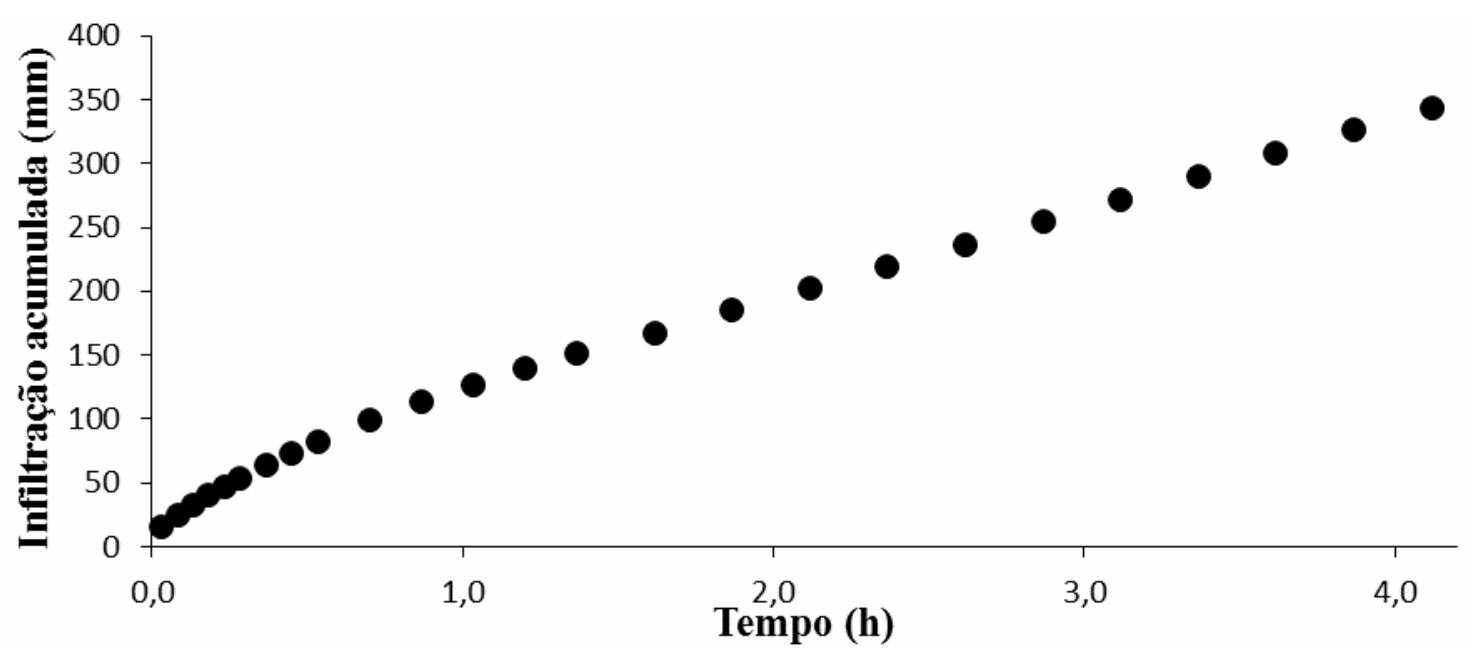

FIGURA 1. Lâmina infiltrada acumulada em função do tempo, pelo ensaio de infiltrômetro de anéis. 


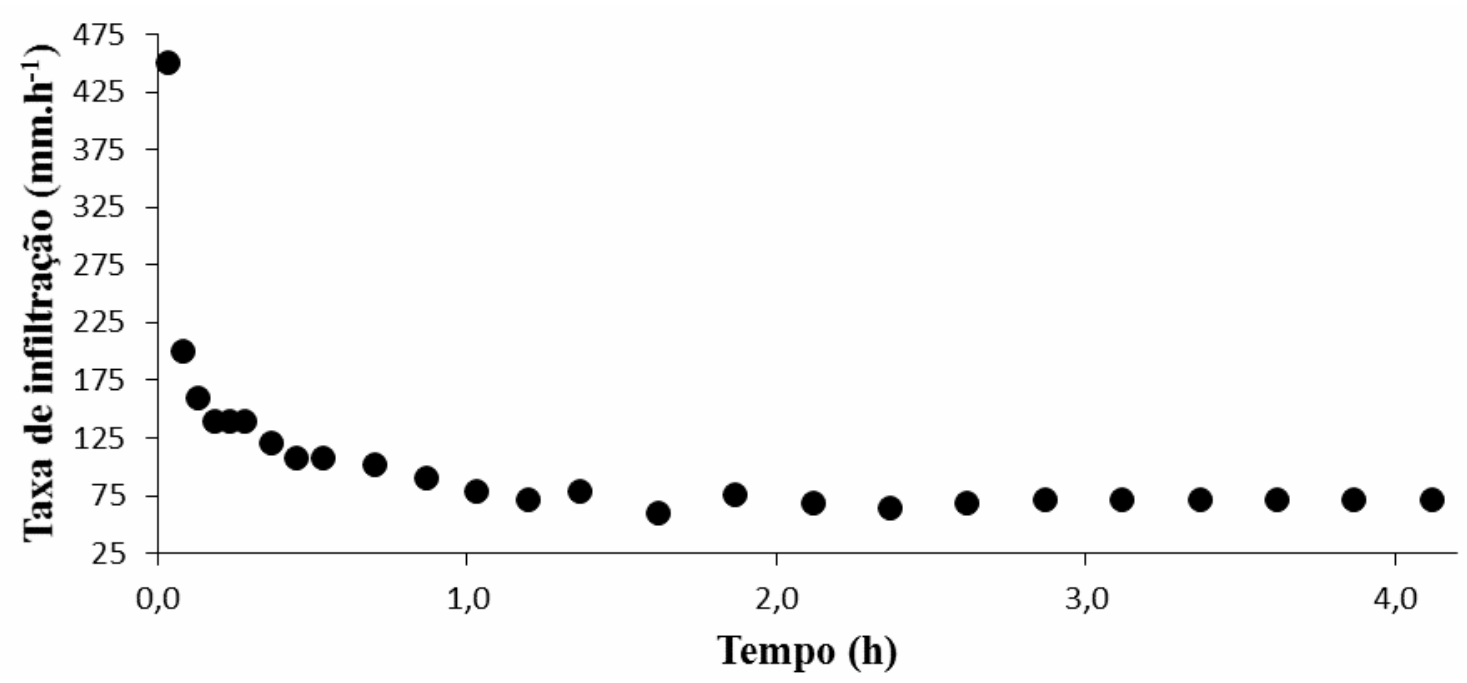

FIGURA 2. Taxa de infiltração da lâmina de água em função do tempo, pelo ensaio de infiltrômetro de anéis.

Em relação aos modelos empíricos de Kostiakov e Kostiakov-Lewis, os pares de valores de lâmina infiltrada e tempo obtidos pelo ensaio de infiltrômetro de anéis, foram utilizados para determinação dos parâmetros das equações dos modelos supra mencionados. Para ajuste, os modelos foram linearizados por meio da aplicação de logaritmos. Para o modelo de Kostiakov, as equações de lâmina infiltrada e taxa de infiltração foram $I=126,3784^{*} t, 6589$ e $i=83,27^{*} t^{0,3411}$, respectivamente. Para o modelo de Kostiakov-Lewis, as equações de lâmina infiltrada e taxa de infiltração foram $I=41,7086^{\star} t^{0,2432}+72^{*} t$ e $i=10,1435^{\star} t^{-0,7568}+$ 72 , respectivamente. As figuras 3 e 4 a seguir apresentam as curvas de lâmina total infiltrada e taxa de infiltração da água no solo, em função do tempo, para os modelos de Kostiakov e Kostiakov-Lewis.

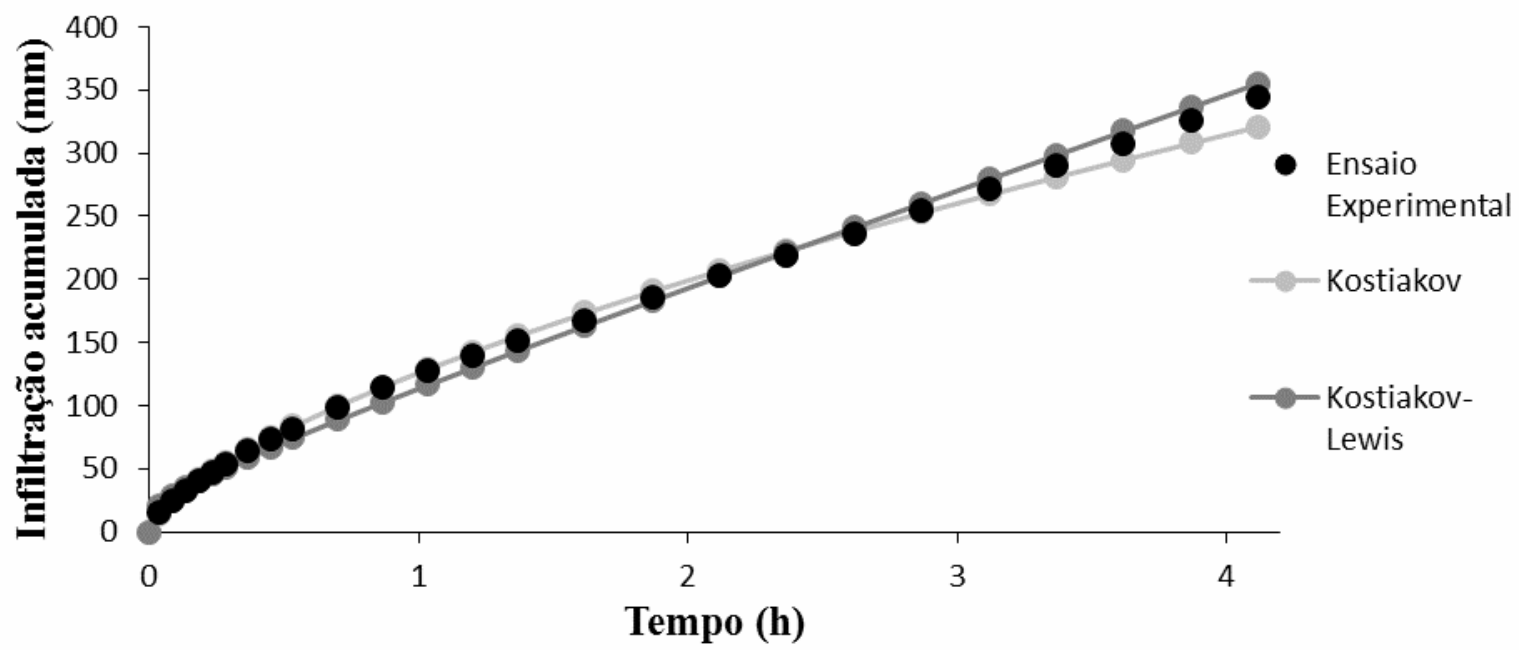

FIGURA 3. Lâmina infiltrada acumulada em função do tempo, pelos modelos de Kostiakov e Kostiakov-Lewis, em comparação aos dados obtidos pelo ensaio de infiltrômetro de anéis. 


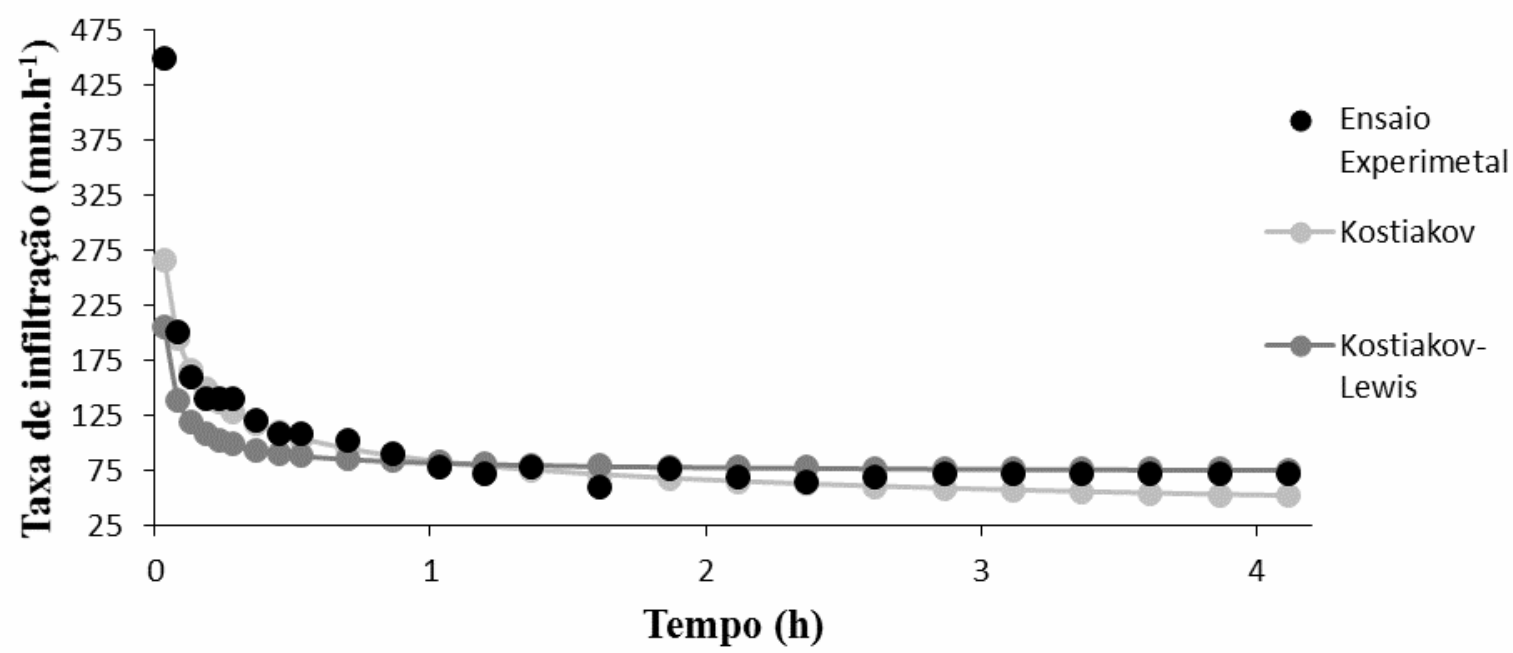

FIGURA 4. Taxa de infiltração da lâmina de água em função do tempo, pelos modelos de Kostiakov e Kostiakov-Lewis, em comparação aos dados obtidos pelo ensaio de infiltrômetro de anéis.

Percebe-se consistência $\left(R^{2}>0,8\right)$ entre as equações ajustadas para os modelos de Kostiakov e Kostiakov-Lewis e os valores obtidos pelo ensaio de infiltrômetro de anéis, sendo que o modelo de Kostiakov-Lewis descreve o processo de infiltração de água no solo de maneira bastante realística, enquanto a análise da curva apresentada pelo modelo de Kostiakov diverge dos resultados obtidos pelo ensaio ao final do mesmo. Resultados semelhantes foram encontrados por NETTO et al. (2013), que ao estudarem o desempenho de modelos de infiltração na região do alto Rio Grande, MG, obtiveram ajustes satisfatórios para previsão da infiltração básica de água no solo pelos modelos de Kostiakov e Kostiakov-Lewis.

Para o modelo de Green-Ampt, os parâmetros obtidos, a partir das amostras de solo coletadas em campo, foram $K_{s}=39,1 \mathrm{~mm} \cdot \mathrm{h}^{-1}, \Psi_{f}=46530,612 \mathrm{~mm}$.c.a., $\theta_{s}=$ $33,42 \%$ e $\theta_{i}=24,14 \%$, enquanto a $T_{i e}$ foi igual a $72 \mathrm{~mm} \cdot \mathrm{h}^{-1}$. CECÍLIO et al. (2013), ao avaliarem a precisão do modelo de Green-Ampt modificado por Mein-Larson, em Latossolo Vermelho-Amarelo, obtiveram, por meio de ensaio com simulador de chuvas, parâmetros $K_{s}$ da magnitude de $112,1 \mathrm{~mm} \cdot \mathrm{h}^{-1}, 129,5 \mathrm{~mm} \cdot \mathrm{h}^{-1}$ e $106 \mathrm{~mm} \cdot \mathrm{h}^{-1}$; $T_{i e}$ da magnitude de $130,3 \mathrm{~mm} \cdot \mathrm{h}^{-1}, 72,5 \mathrm{~mm} \cdot \mathrm{h}^{-1}$ e $107,6 \mathrm{~mm} \cdot \mathrm{h}^{-1} ; \theta_{s}$ da magnitude de $54,10 \%, 53,7 \%$ e $53,4 \%$; e $\theta_{i}$ da magnitude de $32,7 \%$, $32 \%$ e $31,8 \%$, sob condições de precipitação de intensidade de 486, 442 e $398 \mathrm{~mm} \cdot \mathrm{h}^{-1}$, respectivamente. ESPÍRITO SANTO (2011), ao avaliar a condutividade hidráulica saturada na estação experimental da Universidade de Brasília, em Latossolos Vermelho e VermelhoAmarelo, sob condições de pastagem, obteve valor médio para condutividade hidráulica saturada do solo de $597 \mathrm{~mm} \cdot \mathrm{h}^{-1}$, pelo método do infiltrômetro de anéis.

Dentre as combinações apresentadas na tabela 1, aquela que apresentou melhor ajuste aos dados experimentais observados, segundo o RMSE, foi a combinação 13. Assumiu-se, nesta combinação, a condutividade hidráulica do solo como a taxa de infiltração estável, o potencial matricial na frente de umedecimento como $\Psi_{f}=49,03\left(K_{s}+0,02\right)^{-0,4932}$ e $\theta_{w}=0,86 \theta_{g}$. Tais adequações foram consideradas condizentes com o processo de infiltração de água no solo, sob condições de campo e, vai de encontro às pressuposiçõ̃es adotadas para o modelo de Green-Ampt apresentadas por PRUSKI et al. (1997). ZONTA et al. (2010), ao ajustarem o modelo de Green-Ampt para condições de campo, em Cambissolo ENCICLOPÉDIA BIOSFERA, Centro Científico Conhecer - Goiânia, v.11 n.22; p. 9632015 
Háplico Distrófico, na Universidade Federal de Viçosa, obtiveram como melhor combinação para os parâmetros do modelo $\theta_{w}=\theta_{s}, K_{t}=K_{s}$ e, utilizaram uma equação empírica para determinação de $\Psi_{f}$. Estes resultados mostram a grande divergência de resultados que pode ser encontrada em função da pedologia local, mesmo que as áreas estejam localizadas próximas. As figuras 5 e 6 mostram as representações gráficas do modelo de Green-Ampt ajustado para os parâmetros determinados a partir das amostras de solo coletadas e para a combinação $\mathrm{C} 13$, em comparação aos dados observados pelo ensaio de infiltrômetro de anéis, respectivamente.

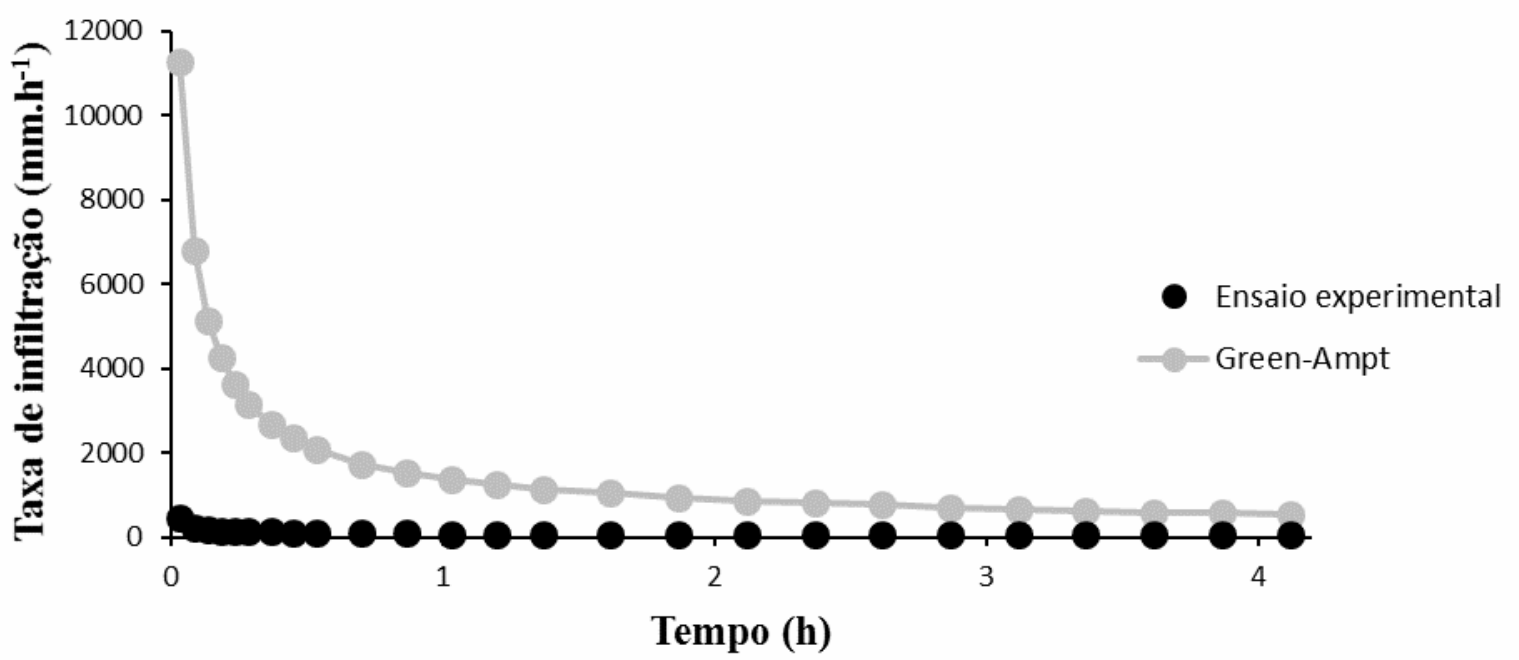

FIGURA 5. Taxa de infiltração da lâmina de água em função do tempo, pelo modelo de Green-Ampt, ajustado aos parâmetros obtidos a partir das amostras de solo, em comparação aos dados obtidos pelo ensaio de infiltrômetro de anéis.

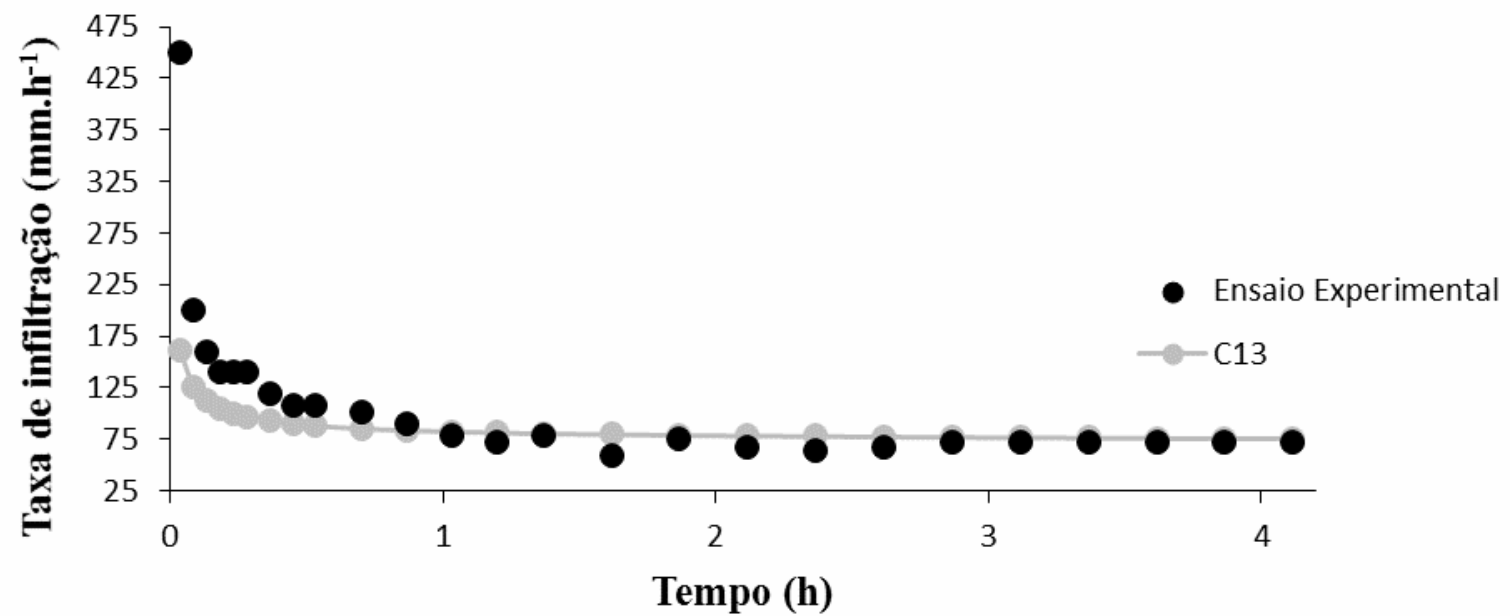

FIGURA 6. Taxa de infiltração da lâmina de água em função do tempo, pelo modelo de Green-Ampt, ajustado aos parâmetros da combinação C13, em comparação aos dados obtidos pelo ensaio de infiltrômetro de anéis. 


\section{CONCLUSÕES}

A partir dos dados obtidos por meio do ensaio de infiltrômetro de anéis foram possíveis os ajustes dos modelos de Kostiakov, Kostiakov-Lewis e Green-Ampt, sendo que os modelos empíricos representaram adequadamente o processo de infiltração de água no solo. Em relação às combinações propostas para o modelo teórico, aquela que melhor se adequou aos dados observados foi a combinação C13, onde os parâmetros assumidos foram $K_{t}=T i e, \theta_{w}=0,86 \theta_{s} \mathrm{e}$ $\Psi_{f}=49,03\left(K_{s}+0,02\right)^{-0,4932}$.

\section{REFERÊNCIAS}

BERNARDO, S. Manual de irrigação. Viçosa: UFV, 1986. 596p.

CECÍLIO, R. A.; SILVA, D. D.; PRUSKI, F. F.; MARTINEZ, M. A. Modelagem da infiltração de água no solo sob condições de estratificação utilizando-se a equação de Green-Ampt. Revista Brasileira de Engenharia Agrícola e Ambiental, v. 7, n. 3, p. 415-422, 2003.

CECÍLIO, R. A.; MARTINEZ, M. A.; PRUSKI, F. F.; SILVA, D. D. Modelo para estimativa da infiltração de água e perfil de umidade do solo. Revista Brasileira de Ciência do Solo, v. 37, p. 411-421, 2013.

EMPRESA BRASILEIRA DE PESQUISA AGROPECUÁRIA - EMBRAPA. Centro Nacional de Pesquisa de Solos. Manual de métodos de análise de solo. 2 e. Rio de Janeiro, 1997. 212p.

ESPÍRITO SANTO, A. F. E. Condutividade hidráulica saturada em função do tipo e uso do solo e método de determinação. Brasília: Unb, 2011. $34 \mathrm{f}$. Dissertação (Mestrado em Agronomia) - Faculdade de Agronomia e Medicina Veterinária, Universidade de Brasília, Brasília, 2011.

GREEN, W. Heber; AMPT, G. A. Studies on soil physics, 1. The flow of air and water through soils. J. Agric. Sci, v. 4, n. 1, p. 1-24, 1911.

KAISER, D. R. Estrutura e água em Argissolo sob distintos preparos na cultura do milho. Santa Maria: UFSM, 2010. 151f. Tese (Doutorado em Ciência do Solo) Centro de Ciências Rurais, Universidade Federal de Santa Maria, Santa Maria, 2010.

KOSTIAKOV, A. N. On the dynamics of the coefficient of water-percolation in soils and on the necessity for studying it from a dynamic point of view for purposes of amelioration. Trans, v. 6, p. 17-21, 1932.

MACHADO, D. F. T.; CONFESSOR, J. G.; RODRIGUES, S. C. Processo inicial de recuperação de área degradada a partir de intervenções físicas e utilização de leguminosas. Caderno de Geografia, v. 24, n. 1, p. 42-54, 2014.

NETTO, G. J. S.; SILVA, A. M.; COELHO, G.; BARROS, D. A.; GUIMARÃES, J. C. C. Desempenho de modelos de infiltração na sub-bacia hidrográfica do ribeirão 
Marcela, na região do alto Rio Grande-MG. Semina: Ciências Agrárias, v. 34, n. 6, p. 2589-2602, 2013.

PRUSKI, F.; SILVA, D., SANTOS, W.; RODRIGUES, L.; ANTUNES, V. Infiltração da água no solo. Viçosa-MG: Engenharia na Agricultura, 1997.

VILARINHO, M. K. C.; KOETZ, M.; SCHLICHTING, A. F.; SILVA, M. C.; BONFIMSILVA, E. M. Determinação da taxa de infiltração estável de água em solo de cerrado nativo. Revista Brasileira de Agricultura Irrigada, v. 7, n. 1, p. 17-26, 2013.

ZONTA, J. H.; MARTINEZ, M. A.; PRUSKI, F. F.; SILVA, D. D.; MONTEBELLER, C. A. Adequação dos parâmetros do modelo de Green-Ampt-Mein-Larson em condições de campo. Revista Brasileira de Engenharia Agrícola e Ambiental, v. 14, n. 10, p. 1021-1029, 2010.

ZONTA, J. H.; MARTINEZ, M. A.; PRUSKI, F. F.; SILVA, D. D.; SANTOS, M. R. Modelagem da infiltração em solos com encrostamento superficial. Parte I: Modelo GAML para solos estratificados. Revista Brasileira de Engenharia Agrícola e Ambiental, v. 16, n. 5, p. 461-470, 2012. 